\title{
Combustion Simulation Using the Lattice Boltzmann Method*
}

\author{
Kazuhiro YAMAMOTO ${ }^{* *}$, Xiaoyi $\mathrm{HE}^{* * *}$ and Gary D. DOOLEN ${ }^{* * * *}$
}

\begin{abstract}
Even though laser diagnostics have significantly improved and can obtain an instantaneous 2D flame image of the velocity field, it is still difficult to obtain data such as scalar flux or reaction rates experimentally. It is also essential to understand 3D flame structures in turbulent combustion. Chemically non-reacting turbulent flows are complex and chemical reactions make the problem more complicated. Due to practical limitations of computational costs, conventional numerical methods are very expensive for carrying out 3D numerical simulations at high Reynolds numbers with detailed chemical reactions. In this study, we have used the lattice Boltzmann method (LBM) to simulate a combustion field. The LBM is an efficient alternative for the numerical simulation of this type of flow. To confirm the validity of the LBM, a flame in simple flow geometry is simulated and the laminar burning velocity is obtained. Both 2D and 3D simulations have been completed. A jet flame has been also simulated to demonstrate the LBM capability of simulating unsteady flames with vortices. The scheme with detailed chemistry has been tested for simulation of a counter-flow flame.
\end{abstract}

Key Words: Computational Fluid Dynamics, Lattice Boltzmann Method, Premixed Combustion, Flame, Chemical Reaction

\section{Introduction}

Even chemically non-reacting turbulent flows are complex phenomena, and chemical reactions make the problem more complicated. Hence, turbulent combustion is very complex phenomena. Laser diagnostics has been largely developed to investigate flame structure with high resolution of time and space. For example, Buschmann et al. ${ }^{(1)}$ have examined the thermal structure by LIF/Rayleigh measurements. Cheng et al. have obtained the flow and scalar fields using PIV/OH-LIF to investigate the turbulence and scalar transport ${ }^{(2)}$. For the present, only 2D image is available, although it is indispensable to discuss a 3D flame structure with turbulence. Also, it is still difficult to estimate some data such as scalar flux or reaction rates experimentally.

Recently, a numerical simulation has been widely

* Received 27th August, 2003 (No. 03-4108)

** Mechanical Engineering, Faculty of Engineering, Nagoya University, Furo-cho, Chikusa-ku, Nagoya-shi, Aichi 464-8603, Japan. E-mail: kazuhiro@mech.nagoyau.ac.jp

*** Computational Modeling Center, Air Products and Chemicals, Inc., 7201 Hamilton Boulevard, Allentown, PA 18195-1501, U.S.A.

**** Theoretical Division, Los Alamos National Laboratory, Los Alamos, NM 87545, U.S.A. used to understand combustion phenomena. Especially, DNS (Direct Numerical Simulation) is powerful means to examine a 3D flame structure. In conventional CFD code, we numerically solve macroscopic continuum equations such as the Navier-Stokes equation. These equations are nonlinear, and enormous small grids and fine time scale are required in combustion simulation. Due to the limitation of computational costs, this method is impractical in carrying out direct 3D simulations with detailed chemistry. Until now, Large-eddy simulation (LES) is realistic system with filtered averaging. In conventional scheme, it is difficult to handle multiphase fluids such as spray combustion, since there are two phases and the assumption of continuity is not valid. Then, an alternative approach may be desirable to simulate combustion in highly turbulent or multiphase flow.

The molecular dynamics (MD) is a more microscopic approach to analyze the phenomena at molecular level. Macroscopic properties such as velocity and temperature are obtained through averaging procedure. Molecular motion is simple at microscopic level. It might be easier to handle fluids, because its dynamical law is linear. However, there are a large number of molecules in real system, and until now, MD is impossible to consider the practical size due to huge computational costs and the lack of memory. 
Recently, the Lattice Boltzmann method (LBM) has been developed into a promising numerical scheme for simulating fluid flows and modeling physics in fluids at mesoscopic level ${ }^{(3)}$. In this study, we conduct some benchmark studies to show the capability of simulating combustion field. For simple case, a flame in uniform flow is focused to obtain the laminar burning velocity. Both 2D and $3 \mathrm{D}$ simulations are conducted. Also, to handle the unsteady flame behavior with vortex, a jet flame is simulated. The scheme with detailed chemistry is tested for simulation of a counter-flow flame.

\section{Lattice Boltzmann Method (LBM)}

In this section, we explain the strategy of the lattice Boltzmann method. The fundamental idea of the LBM is to construct simplified kinetic models that incorporate the essential physics of microscopic or mesoscopic processes so that the macroscopic averaged properties obey the desired macroscopic equations such as the N-S equation. The kinetic equation provides any of the advantages of molecular dynamics, including clear physical pictures, easy implementation of boundary conditions, and fully parallel algorisms ${ }^{(3)}$. The LBM fulfills these requirements in a straightforward manner.

So far, many studies have been conducted. For example, He and Doolen have simulated the flow around twodimensional circular cylinder to show the time evolution of vortex shedding ${ }^{(4)}$. Martinez et al. have examined the turbulence in shear layer, and the turbulent flow can be well simulated at a relatively high Reynolds number of $10000^{(5)}$. Compared with conventional methods, the lattice Boltzmann scheme is simple for handling boundary conditions. Flow in complex geometries has been simulated such as fluids in porous media ${ }^{(6)}$. Also, simulations of multiphase flow have been conducted ${ }^{(7)}$. The special treatment is not needed and each phase can be treated equally. LBM could be a better tool for many combustion applications including spray combustion.

In a recent study, we have proposed an approach to simulate combustion field by $\mathrm{LBM}^{(8)}$. The model is composed of LB equations for flow, temperature and mass fraction of species, which is explained in the next section. This is the first LB simulation on combustion, although coupled approach has been proposed to simulate flow field by LBM and temperature and concentration fields by a finite difference scheme ${ }^{(9)}$. Figure 1 shows the reaction distribution in previous simulation of a counterflow flame. The inlet velocity, $U_{0}$, at the wall is $0.2 \mathrm{~m} / \mathrm{s}$ with $L=10 \mathrm{~mm}$. The equivalence ratio of propane/air

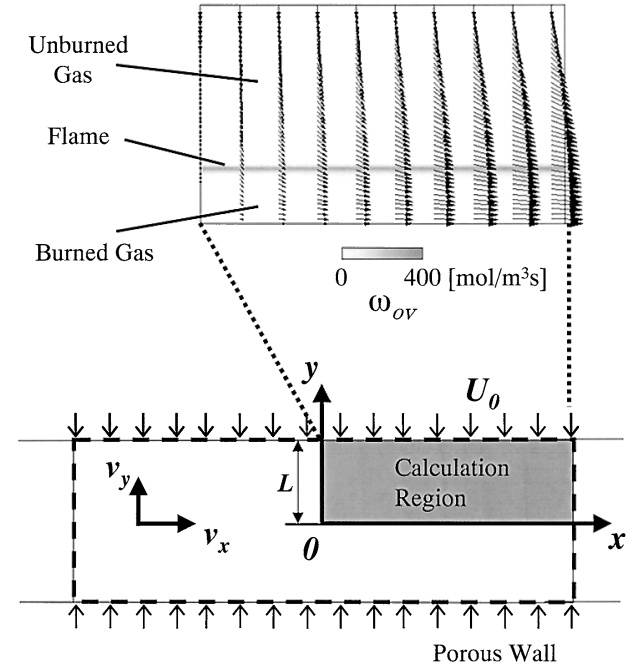

Fig. 1 Flame and flow configurations; $\phi=0.6$

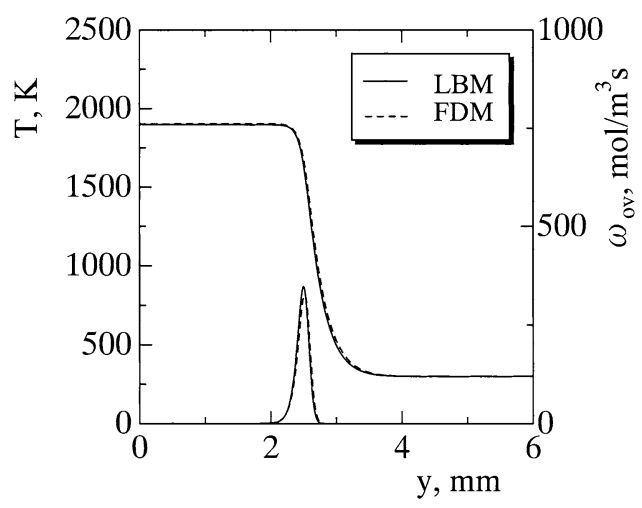

Fig. 2 Distributions of temperatures and over-all reaction rate for propane/air mixtures; $\phi=0.6$

mixtures is 0.6 and its Reynolds number $\left(=U_{0} L / v\right)$ is 124. From this figure, the counter-flow flame structure is well simulated. To validate the LB simulation, we have compared our results with those by conventional method, solving differential conservation equations of mass, momentum, energy, and species by a finite difference method (FDM). The comparison is shown in Fig. 2, with the distributions of temperature and over-all reaction rate. Both results are perfectly matched. We have concluded that LBM can be used for combustion simulation.

\section{LB Equations and Boundary Conditions}

Here, we explain the numerical procedure in detail. The schematic plot of velocity directions is shown in Fig. 3. The following square lattice $\left(\mathrm{d} 2 \mathrm{q} 9\right.$ model $\left.^{(10)}\right)$ is used in 2D simulation, which is seen in Fig. 3 (a):

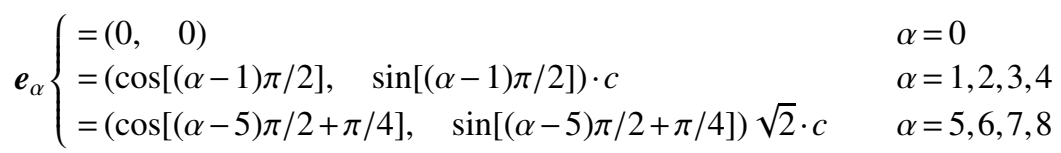




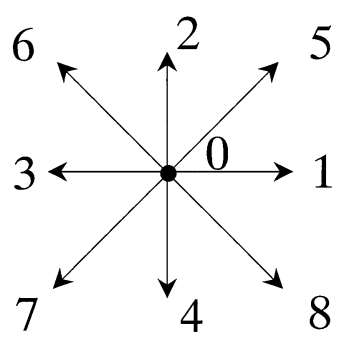

(a) 2D model (d2q9)



(b) 3D model (d3q15)

Fig. 3 Schematic plot of velocity directions of 2D and 3D models in LBM

where $c$ is the advection speed. For the incompressible fluid, the evolution equation using the pressure distribution function is

$$
p_{\alpha}\left(\boldsymbol{x}+\boldsymbol{e}_{\alpha} \delta_{t}, t+\delta_{t}\right)-p_{\alpha}(\boldsymbol{x}, t)=-\left[p_{\alpha}(\boldsymbol{x}, t)-p_{\alpha}^{e q}(\boldsymbol{x}, t)\right] / \tau
$$

where $\delta_{t}$ is the time step, and $\tau$ is the relaxation time which controls the rate of approach to equilibrium. It is proved that the LBM is just one of the discretized form of the Boltzmann equation. A rigorous derivation of the LBM from the kinetic theory has been already shown ${ }^{(11)}$. The equilibrium distribution function, $p_{\alpha}{ }^{e q}$, is given by

$$
\begin{aligned}
& p_{\alpha}{ }^{e q}(\boldsymbol{x}, t) \\
& =w_{\alpha}\left\{p+p_{0}\left[3\left(\boldsymbol{e}_{\alpha} \cdot \boldsymbol{u}\right) / c^{2}+9\left(\boldsymbol{e}_{\alpha} \cdot \boldsymbol{u}\right)^{2} / 2 / c^{4}-3 \boldsymbol{u} \cdot \boldsymbol{u} / 2 / c^{2}\right]\right\}
\end{aligned}
$$

where $w_{0}=4 / 9, w_{\alpha}=1 / 9(\alpha=1: 4), w_{\alpha}=1 / 36(\alpha=5: 8)$. The sound speed, $c_{s}$, is $c / \sqrt{3}$ for $\mathrm{d} 2 \mathrm{q} 9$ model and $c / \sqrt{(8 / 3)}$ for $\mathrm{d} 3 \mathrm{q} 15$ model, with $p_{0}=\rho_{0} c_{s}^{2}$. The pressure, $p$, and the velocity, $\boldsymbol{u}=\left(V_{x}, V_{y}\right)$, are calculated by

$$
\begin{aligned}
& p=\Sigma p_{\alpha} \\
& \boldsymbol{u}=\Sigma \boldsymbol{e}_{\alpha} p_{\alpha} / p_{0}
\end{aligned}
$$

In a compressible model for variable density, the distribution function of density is used ${ }^{(12)}$. Through the Chapman-Enskog procedure, the Navier-Stokes equations are derived from the equations for density distribution functions ${ }^{(10)}$. The relaxation time is related with transport coefficients, such as kinetic viscosity and diffusion coefficient. The kinetic viscosity is

$$
v=(2 \tau-1) / 6 \times \delta_{x}^{2} / \delta_{t}
$$

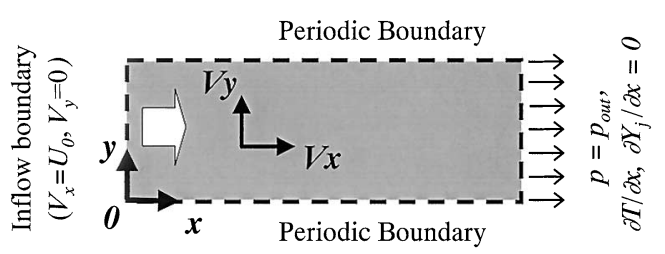

(a) Laminar flame in uniform flow

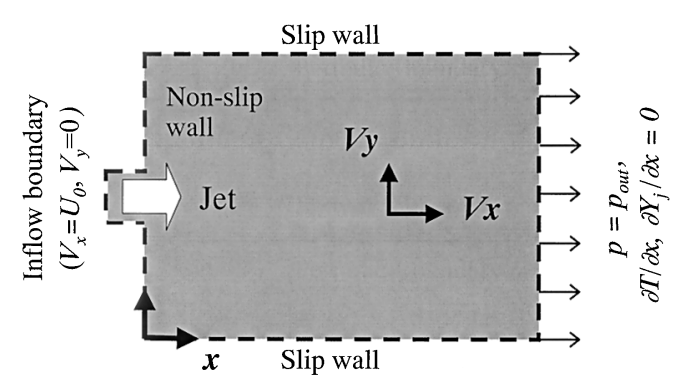

(b) Turbulent jet flame

Fig. 4 Coordinate and boundary condition

For 3D simulation, the $\mathrm{d} 3 \mathrm{q} 15$ model in Fig. $3(\mathrm{~b})$ is used $^{(13)}$. It may not be needed when we obtain a laminar burning velocity, but 3D model is applied to show the example of 3D simulation. LB formula for temperature and concentration fields is as follows:

$$
\begin{aligned}
& F_{s, \alpha}\left(\boldsymbol{x}+\boldsymbol{e}_{\alpha} \delta_{t}, t+\delta_{t}\right)-F_{s, \alpha}(\boldsymbol{x}, t) \\
& \quad=-\left[F_{s, \alpha}(\boldsymbol{x}, t)-F_{s, \alpha}^{e q}(\boldsymbol{x}, t)\right] / \tau_{s}+w_{\alpha} Q_{s} \\
& F_{s, \alpha}{ }^{e q}(\boldsymbol{x}, t) \\
& \quad=w_{\alpha} s\left\{1+3\left(\boldsymbol{e}_{\alpha} \cdot \boldsymbol{u}\right) / c^{2}+9\left(\boldsymbol{e}_{\alpha} \cdot \boldsymbol{u}\right)^{2} / 2 / c^{4}-3 \boldsymbol{u} \cdot \boldsymbol{u} / 2 / c^{2}\right\} \\
& s=T, Y_{i} .
\end{aligned}
$$

The temperature, $T$, and mass fraction of species, $Y_{i}$, are obtained in terms of the distribution function by

$$
\begin{aligned}
& T=\Sigma F_{T, \alpha} \\
& Y_{i}=\Sigma F_{Y i, \alpha} .
\end{aligned}
$$

The source term due to chemical reaction, $Q_{s}$, is given by the similarity in non-dimensional forms of LB coordinate and real coordinate. The fuel used is propane for the flame in uniform flow and the jet flame. The following over-all reaction rate $\left[\mathrm{mol} / \mathrm{m}^{3} \mathrm{~s}\right]$ is adopted.

$$
\omega_{o v}=k_{o v} \mathrm{C}_{\mathrm{C}_{3} \mathrm{H}_{8}}{ }^{a} \mathrm{C}_{\mathrm{O}_{2}}{ }^{b} \exp (-E / R T)
$$

All parameters including reaction coefficient and effective activation energy are referred to Ref.(14). The reaction coefficient, $k_{o v}$, is $8.6 \times 10^{11}\left[\mathrm{~cm}^{3} / \mathrm{mol}\right]$, effective activation energy, $E$, is 30 [kcal $/ \mathrm{mol}]$, and reaction coefficients of $a$ and $b$ are 0.1 and 1.65 , respectively. Hydrogen/air mixture is used for the counter-flow flame with detailed chemistry.

Figure 4 shows the coordinate and boundary conditions in this calculation. For simulation of a flame in uniform flow, two-dimensional rectangular coordinates are used for channel length of $10 \mathrm{~cm}$. The flame is formed in uniform flow with constant velocity, $U_{0}$. The flame 
propagates in the flow direction ( $x$-direction) like a onedimensional planar wave, if the high temperature region is given to ignite the mixture. Then, we estimate the laminar burning velocity. As seen in Fig. 4(a), at the inlet, inflow boundary for flow field ${ }^{(12)}$ and hydrodynamic conditions for the temperature and concentration are used ${ }^{(15)}$. Both upper and lower boundaries have periodic boundary conditions, obtaining the uniform flow field in the whole domain. At the exit, developed boundary condition is adopted. We have conducted convergence study, changing grid points in $N_{x}$ in $x$-direction and $N_{y}$ in $y$-direction ${ }^{(16)}$. When $N_{y} \geq 41$, the simulation result is converged to obtain the same burning velocity. When $N_{x}$ is varied, the number of grid points describing the reaction region is changed. For example, there are only 3 points for $N_{x}=600$, and about 10 points for $N_{x}=2000$ when the equivalence ratio is unity. For $N_{x}>1200$, the same flame structure such as temperature and concentration profiles is observed. Here, we use the grid points of $3001\left(N_{x}\right) \times 41\left(N_{y}\right)$ for $2 \mathrm{D}$ calculation, and $3001\left(N_{x}\right) \times 41\left(N_{y}\right) \times 41\left(N_{z}\right)$ for 3D calculation.

The jet flame is also simulated. As seen in Fig. 4 (b), inflow boundary condition is used at the inlet. The nonslip boundary condition is adopted at walls of the jet nozzle. At both upper and lower boundaries, the slip wall boundary is used. The number of grid points is $241\left(N_{x}\right) \times 225\left(N_{y}\right)$. The domain size is large enough to avoid the boundary effect.

\section{Results and Discussion}

\subsection{Flame in uniform flow}

First, the result of the flame in uniform flow is shown. The inlet velocity of a propane/air mixture, $U_{0}$, is $1 \mathrm{~m} / \mathrm{s}$ and its temperature, $T_{0}$, is room temperature $(=300 \mathrm{~K})$. Figure 5 shows the temperature and concentration distributions. The equivalence ratio is 1.0. It should be noted that, in simulations, parameters are calculated in nondimensional form, and results are transferred to have the real unit based on the similarity. The flame front is flat like a one-dimensional flame, and the profiles across the flame are the same. As seen in Fig. 5(a), the temperature starts to increase at $x=21 \mathrm{~mm}$, and the reaction zone is located at $x=22 \mathrm{~mm}$, where the large heat release occurs. Temperature becomes constant in the burned gas region. Figure 5 (b) shows the mass fraction profiles. The reactants, $\mathrm{C}_{3} \mathrm{H}_{8}$ and $\mathrm{O}_{2}$, begin decreasing at the edge of preheat zone $(x=21 \mathrm{~mm})$, and react in the reaction zone to become products, $\mathrm{CO}_{2}$ and $\mathrm{H}_{2} \mathrm{O}$. Then, the fine flame structure is observed.

Next, we obtain the laminar burning velocity, $S_{L}$. In this simulation, as the inlet velocity is always larger than $S_{L}$, the flame moves downstream. Since the flame propagation speed, $V_{f}$, is equal to the inlet velocity subtracted by the burning velocity, the burning velocity can be estimated by $S_{L}=U_{0}-V_{f}$. The obtained burning velocity



Fig. 5 Distributions of (a) temperature and over-all reaction rate, (b) mass fraction of species; $\phi=1.0$



Fig. 6 Variations of burning velocity with equivalence ratio

is shown in Fig. 6. It is found that both results of 2D and 3D simulations are almost the same. Compared with the experimental value by Yamaoka and Tsuji ${ }^{(17)}$, these results show relatively good agreement, although the calculated value slightly overestimates $S_{L}$. This may be because our scheme is based on an incompressible model with an over-all one step reaction ${ }^{(16)}$. However, based on numerical simulation with GRI-Mech 3.0 for a propane/air mixture $^{(18)}$, the calculated burning velocity is larger than experimental data. Then, it is confirmed that the combustion field is well simulated by our proposed model.

\subsection{Jet flame}

To show the capability of handling the unsteady flame behavior in LB simulation, a jet flame is also simulated. The inlet velocity is $5 \mathrm{~m} / \mathrm{s}$, and the nozzle diameter is $6 \mathrm{~mm}$. The equivalence ratio is 0.6 , and its Reynolds number is 1900 . Hence, its condition is for a 2D transitional jet.

The mixture is ejected to the stationary air, and the vortex is formed due to the shear stress. Figure 7 shows the vorticity distributions in turbulent jet flames. The vorticity is defined by $\omega=\partial V_{y} / \partial x-\partial V_{x} / \partial y$. The velocity vector is 

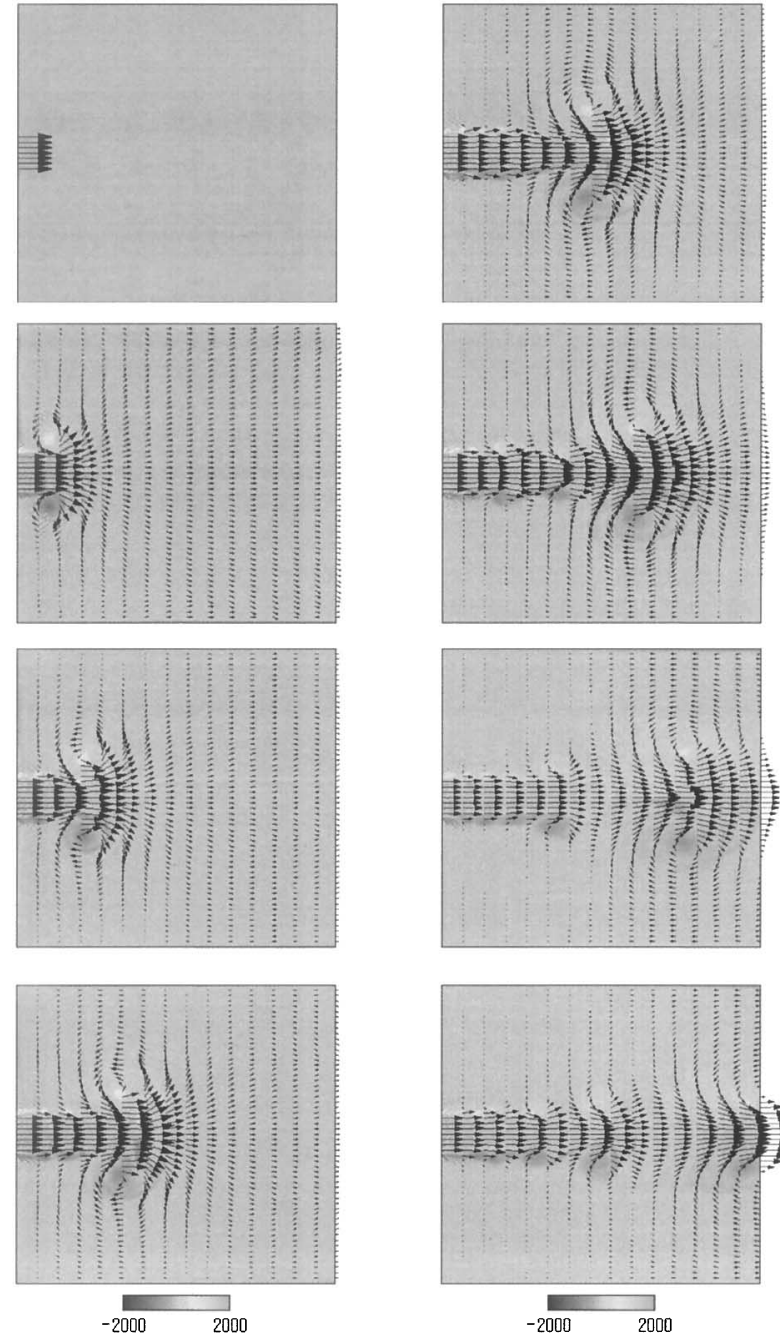

$\omega[1 / \mathrm{s}]$
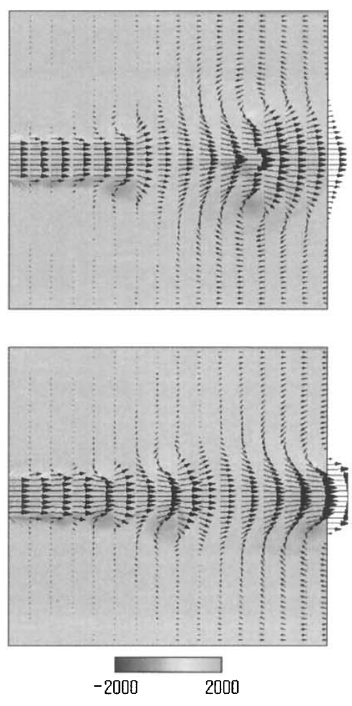

$\omega[1 / \mathrm{s}]$

Fig. 7 Vorticity distributions observed in turbulent jet flame at $R e=1900$ by LBM; time step $=0,1000,2000,3000$, $4000,5000,6000$, and 7000 (from left top to right bottom)

also shown. The vortex structure is developed as the time step goes. Figure 8 shows the distributions of vorticity and temperature. It is found that the flame structure is largely affected by the vortex, and the flame is always fluctuating. Although the interaction between flame and eddies is not discussed here, LBM can be used to simulate the turbulent combustion.

\subsection{Counter-flow flame with detailed chemistry}

Finally, the scheme with detailed chemistry is tested. Here, a counter-flow flame is simulated, which is formed in hydrogen/air mixtures. The 13-step chemical-kinetic scheme is used. The mechanism consists of 20 elementary steps among 8 reacting species and nitrogen. The specific reaction-rate constants for forward and backward reactions are shown in Table $1^{(19)-(21)}$. The coordinate and grid numbers are the same as those in previous study ${ }^{(8)}$.


(a) Vorticity, $\omega[1 / \mathrm{s}]$

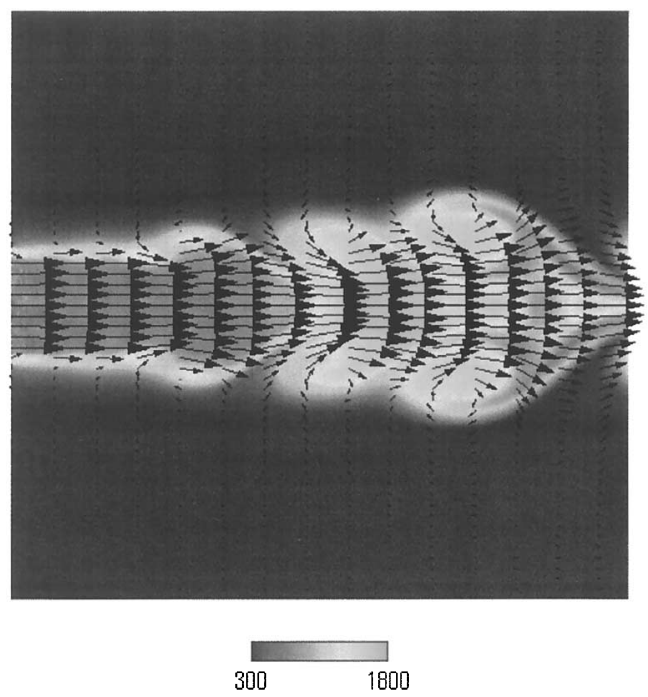

(b) Temperature, $T[\mathrm{~K}]$

Fig. 8 Distributions of vorticity and temperature, time step $=$ 12000

Figure 9 shows the distributions of temperature and heat release rate for $\phi=0.18$. The porous wall is located at $y=10 \mathrm{~mm}$. As the center is approached, the temperature starts to increase at $y=5 \mathrm{~mm}$, steeply increases around the reaction zone where the large heat release occurs, and takes its maximum at $y=0 \mathrm{~mm}$.

Figure 10 shows the mass fraction profile for reactive species. As seen in this figure, $Y_{\mathrm{H}_{2}}$ and $Y_{\mathrm{O}_{2}}$ start to decrease at the edge of preheat zone $(y=3-5 \mathrm{~mm})$, and then $\mathrm{H}_{2}$ and $\mathrm{O}_{2}$ react in the reaction zone to produce the product, $\mathrm{H}_{2} \mathrm{O}$. The mass fraction of $\mathrm{OH}, \mathrm{O}, \mathrm{H}$ is very small compared with those of major species, $\mathrm{H}_{2}, \mathrm{O}_{2}$ and $\mathrm{H}_{2} \mathrm{O}$. These radicals play important roles on combustion processes, and mainly exist in the reaction region. On the other hand, hydrogen peroxide $\mathrm{H}_{2} \mathrm{O}_{2}$ and hydroperoxyl radical $\mathrm{HO}_{2}$ exist in a considerably broad region. This 
Table 1 Specific reaction-rate constants for the reaction scheme

\begin{tabular}{|c|c|c|c|c|}
\hline $\mathrm{NO}$ & Reaction & A & $\alpha$ & E \\
\hline if & $\mathrm{H}+\mathrm{O}_{2} \rightarrow \mathrm{OH}+\mathrm{O}$ & $2.00 \mathrm{E} 14$ & 0.00 & 70.30 \\
\hline $1 \mathrm{~b}$ & $\mathrm{OH}+\mathrm{O} \rightarrow \mathrm{H}+\mathrm{O}_{2}$ & $1.57 \mathrm{E} 13$ & 0.00 & 2.89 \\
\hline $2 \mathrm{f}$ & $\mathrm{H}_{2}+\mathrm{O} \rightarrow \mathrm{OH}+\mathrm{H}$ & 1.80E10 & 1.00 & 36.93 \\
\hline $2 \mathrm{~b}$ & $\mathrm{OH}+\mathrm{H} \rightarrow \mathrm{H}_{2}+\mathrm{O}$ & $8.00 \mathrm{E} 09$ & 1.00 & 28.29 \\
\hline $3 \mathrm{f}$ & $\mathrm{H}_{2} \mathrm{O}+\mathrm{O} \rightarrow \mathrm{OH}+\mathrm{OH}$ & $5.90 \mathrm{E} 09$ & 1.30 & 71.25 \\
\hline $3 b$ & $\mathrm{OH}+\mathrm{OH} \rightarrow \mathrm{H}_{2} \mathrm{O}+\mathrm{O}$ & $6.00 \mathrm{E} 08$ & 1.30 & 0.00 \\
\hline $4 \mathrm{f}$ & $\mathrm{H}_{2}+\mathrm{OH} \rightarrow \mathrm{H}_{2} \mathrm{O}+\mathrm{H}$ & $1.17 \mathrm{E} 09$ & 1.30 & 15.17 \\
\hline $4 \mathrm{~b}$ & $\mathrm{H}_{2} \mathrm{O}+\mathrm{H} \rightarrow \mathrm{H}_{2}+\mathrm{OH}$ & 5.09E09 & 1.30 & 77.78 \\
\hline 5 & $\mathrm{H}+\mathrm{O}_{2}+\mathrm{M} \rightarrow \mathrm{HO}_{2}+\mathrm{M}$ & $2.30 \mathrm{E} 18$ & -0.80 & 0.00 \\
\hline 6 & $\mathrm{H}+\mathrm{HO}_{2} \rightarrow \mathrm{OH}+\mathrm{OH}$ & $1.50 \mathrm{E} 14$ & 0.00 & 4.20 \\
\hline $7 \mathrm{f}$ & $\mathrm{H}+\mathrm{HO}_{2} \rightarrow \mathrm{H}_{2}+\mathrm{O}_{2}$ & $2.50 \mathrm{E} 13$ & 0.00 & 2.93 \\
\hline $7 \mathrm{~b}$ & $\mathrm{H}_{2}+\mathrm{O}_{2} \rightarrow \mathrm{H}+\mathrm{HO}_{2}$ & 7.27E13 & 0.00 & 244.33 \\
\hline 8 & $\mathrm{OH}+\mathrm{HO}_{2} \rightarrow \mathrm{H}_{2} \mathrm{O}+\mathrm{O}_{2}$ & $2.00 \mathrm{E} 13$ & 0.00 & 4.18 \\
\hline 9 & $\mathrm{H}+\mathrm{H}+\mathrm{M} \rightarrow \mathrm{H}_{2}+\mathrm{M}$ & $1.80 \mathrm{E} 18$ & -1.0 & 0.00 \\
\hline 10 & $\mathrm{H}+\mathrm{OH}+\mathrm{M} \rightarrow \mathrm{H}_{2} \mathrm{O}+\mathrm{M}$ & $2.20 \mathrm{E} 22$ & -2.0 & 0.00 \\
\hline 11 & $\mathrm{HO}_{2}+\mathrm{HO}_{2} \rightarrow \mathrm{H}_{2} \mathrm{O}_{2}+\mathrm{O}_{2}$ & $2.00 \mathrm{E} 12$ & 0.00 & 0.00 \\
\hline $12 f$ & $\mathrm{H}_{2} \mathrm{O}_{2}+\mathrm{M} \rightarrow \mathrm{OH}+\mathrm{OH}+\mathrm{M}$ & $1.30 \mathrm{E} 17$ & 0.00 & 190.38 \\
\hline $12 \mathrm{~b}$ & $\mathrm{OH}+\mathrm{OH}+\mathrm{M} \rightarrow \mathrm{H}_{2} \mathrm{O}_{2}+\mathrm{M}$ & 9.86Е14 & 0.00 & -21.22 \\
\hline $13 \mathrm{f}$ & $\mathrm{H}_{2} \mathrm{O}_{2}+\mathrm{OH} \rightarrow \mathrm{H}_{2} \mathrm{O}+\mathrm{HO}_{2}$ & $1.00 \mathrm{E} 13$ & 0.00 & 7.53 \\
\hline $13 \mathrm{~b}$ & $\mathrm{H}_{2} \mathrm{O}+\mathrm{HO}_{2} \rightarrow \mathrm{H}_{2} \mathrm{O}_{2}+\mathrm{OH}$ & $2.86 \mathrm{E} 13$ & 0.00 & 137.21 \\
\hline
\end{tabular}

The reaction constants are written as $k=A T^{\alpha} \exp \left(-E / R_{u} T\right)$, with the individual quantities expressed in $\mathrm{cm}, \mathrm{mol}, \mathrm{s}, \mathrm{kJ}$ and $K$ units.

The third-body efficiencies are 0.40 for $\mathrm{O}_{2}, 6.50$ for $\mathrm{H}_{2} \mathrm{O}, 0.40$ for $\mathrm{N}_{2}$, and 1.00 for all other species.



Fig. 9 Distributions of temperature and heat release rate for hydrogen/air mixtures; $\phi=0.18$

is because these radicals are thought to be relatively stable species. From these figures, the fine flame structure is well simulated with detailed chemistry.

\section{Conclusions}

In this present paper, we have simulated combustion field using the Lattice Boltzmann method. Both 2D and 3D models are used. The schemes with overall onestep and detailed reactions have been tested. We have focused on very simple case of flames in uniform and counter flows. To confirm the validity of LB simulation,

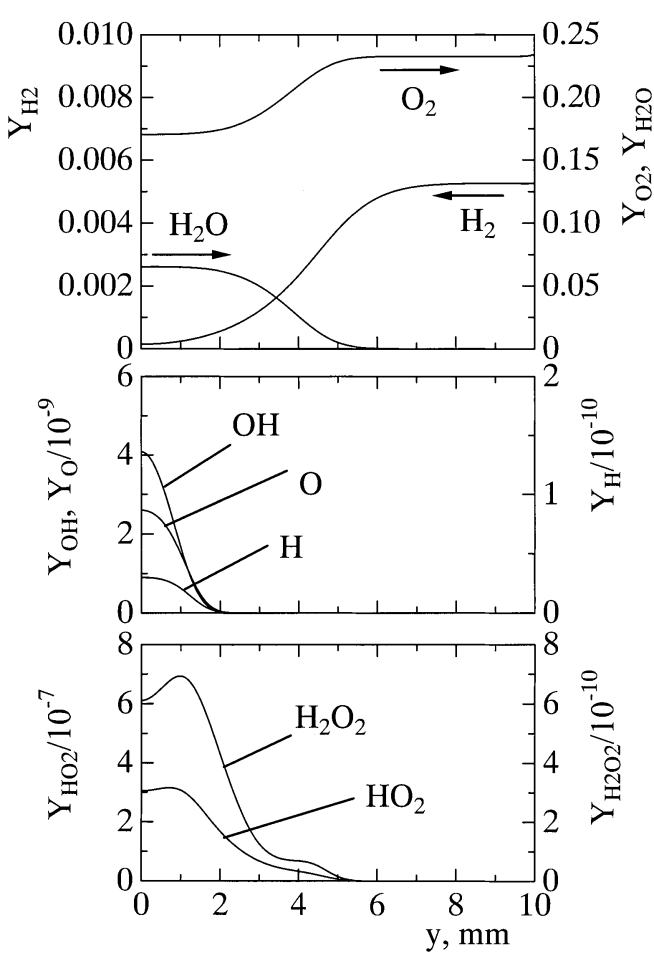

Fig. 10 Mass fraction distributions for hydrogen/air mixtures; $\phi=0.18$

we have estimated a laminar burning velocity, compared with experimental values. Simulation results show relatively good agreement with those by Tsuji et al. The jet flame has been also simulated to show the capability of handling the unsteady flame behavior. The flame structure affected by the vortex is well described. Hence, it is demonstrated that the Lattice Boltzmann method can be an alternative approach for combustion simulation with turbulence.

\section{Acknowledgements}

This work was partly supported by Grant-in-Aid for Young Scientists (B) No.13 750 170, from Japan Society for the Promotion of Science.

\section{References}

( 1 ) Buschmann, A., Dinkelacker, F., Schafer, T. and Wolfrum, J., Proc. Comb. Inst., Vol.26, 437 (1996).

(2) Cheng, Y.C. and Bilger, R.W., Proc. Comb. Inst., Vol.28, 521 (2000).

( 3 ) Chen, S. and Doolen, G.D., Annual Reviews of Fluid Mech., Vol.30 (1998), pp.329-364.

( 4 ) He, X. and Doolen, G.D., Physical Review, E56-1 (1997), pp.434-440.

( 5 ) Martinez, D.O., Matthaeus, W.H., Chen, S. and Montgomery, D.C., Phys. Fluids, Vol.6 (1994), pp.1285-1298.

( 6 ) Succi, S., Foti, E. and Higuera, F., Euro-phys. Lett., Vol.10, 433 (1989).

( 7 ) He, X., Shan, X.Y. and Doolen, G.D., Phys. Rev. E., Vol.57, R13 (1998). 
( 8 ) Yamamoto, K., He, X. and Doolen, G.D., J. Statistical Physics, Vol.7, Nos.1/2 (2002), pp.367-383.

(9) Filippova, O. and Hanel, D., J. Comput. Physics, Vol.158 (2000), pp.139-160.

(10) He, X. and Luo, L.S., J. Statistical Physics, Vol.88, Nos. 3/4 (1997), pp.927-944.

(11) He, X. and Luo, L.S., Phys. Rev. E, Vol.56 (1997), pp.6811-6817.

(12) Zou, Q. and He, X., Phys. Fluids, Vol.9, No.6 (1997), pp.1591-1598.

(13) Qian, Y.H., d'Humieres, D. and Lallemand, P., Europhys. Lett., Vol.17 (1992), pp.479-484.

(14) Westbrook, C.K. and Dryer, F.L., Combust. Sci. Technol., Vol.27 (1981), pp.31-43.

(15) He, X., Chen, S. and Doolen, G.D., J. Comput. Phys.,
Vol.146 (1998), pp.282-300.

(16) Yamamoto, K., Journal of Mechanical Engineering (B), (in Japanese), Vol.68-674 (2002), pp.2908-2913.

(17) Yamaoka, I. and Tsuji, H., Proc. Comb. Inst., Vol.20, 1883 (1984).

(18) http://www.me.berkeley.edu/gri_mech/version30/ figs30/C3Su.gif

(19) Rogg, B., Lecture Notes in Physics, Vol.384 (1991), pp.159-192.

(20) Gutheil, E., et al., Reduced Kinetic Mechanism for Applications in Combustion Systems, (1993), pp.177195.

(21) Sanchez, A.L., Balakrishnan, G., Linan, A. and Williams, F.A., Combust. Flame, Vol.87 (1996), pp.569-590. 